\title{
An all-sky proper-motion map of the Sagittarius stream using Gaia DR2 ${ }^{\star}$
}

\author{
T. Antoja ${ }^{1}$, P. Ramos ${ }^{1}$, C. Mateu ${ }^{2}$, A. Helmi ${ }^{3}$, F. Anders ${ }^{1}$, C. Jordi ${ }^{1}$, and J. A. Carballo-Bello ${ }^{4}$ \\ 1 Institut de Ciències del Cosmos, Universitat de Barcelona (IEEC-UB), Martíi Franquès 1, 08028 Barcelona, Spain \\ e-mail: tantoja@fqa.ub.edu \\ 2 Departamento de Astronomía, Facultad de Ciencias, Universidad de la República, Iguá 4225, 14000 Montevideo, Uruguay \\ 3 Kapteyn Astronomical Institute, University of Groningen, 9747 AD Groningen, The Netherlands \\ ${ }^{4}$ Instituto de Astrofísica, Pontificia Universidad Católica de Chile, Av. Vicuña Mackenna 4860, 782-0436 Macul, Santiago, Chile
}

Received 19 November 2019 / Accepted 31 January 2020

\begin{abstract}
Aims. We aim to measure the proper motion along the Sagittarius stream, which is the missing piece in determining its full 6D phase space coordinates.

Methods. We conduct a blind search of over-densities in proper motion from the Gaia second data release in a broad region around the Sagittarius stream by applying wavelet transform techniques.

Results. We find that for most of the sky patches, the highest intensity peaks delineate the path of the Sagittarius stream. The 1500 peaks identified depict a continuous sequence spanning almost $2 \pi$ in the sky, only obscured when the stream crosses the Galactic disk. Altogether, around 100000 stars potentially belong to the stream as indicated by a coarse inspection of the color-magnitude diagrams. From these stars, we determine the proper motion along the Sagittarius stream, making it the proper-motion sequence with the largest span and continuity ever measured for a stream. A first comparison with existing $N$-body models of the stream reveals some discrepancies, especially near the pericenter of the trailing arm and an underestimation of the total proper motion for the leading arm.

Conclusions. Our study provides a starting point for determining the variation of the population of stars along the stream, the distance to the stream from the red clump stars, and the solar motion. It also permits much more accurate measurement of the Milky Way potential.
\end{abstract}

Key words. Galaxy: halo - galaxies: dwarf - Galaxy: kinematics and dynamics - Galaxy: formation - astrometry

\section{Introduction}

Research into tidal streams in the Milky Way has already benefited immensely from the Gaia mission (Gaia Collaboration 2016). The outstanding and precise proper motions of the second data release (DR2; Gaia Collaboration 2018) fostered the discovery of many new streams, both with known (Ibata et al. 2019a; Palau \& Miralda-Escudé 2019) and unknown (Ibata et al. 2018, 2019b; Malhan et al. 2018a) progenitors, and mapping over larger portions of the sky (Price-Whelan \& Bonaca 2018; Koposov et al. 2019). Gaia has also been crucial for the detection of peculiarities in streams, such as diffuse components and gaps, which could provide information about the structure of the progenitor and of the Milky Way halo, and about the dark subhalos that could have interacted with the stream stars (e.g., Bonaca et al. 2019a,b; Malhan et al. 2018b, 2019; Price-Whelan \& Bonaca 2018). The Gaia DR2 has also revealed evidence of misaligned proper motions, which seem to be a telltale sign of the gravitational effects of other Milky Way massive satellites (Koposov et al. 2019; Erkal et al. 2019; Shipp et al. 2019).

The stream of the Sagittarius dwarf (Sgr, Ibata et al. 1994) discovered by e.g. Mateo et al. (1996) and Totten \& Irwin (1998)

\footnotetext{
* Full Table E.1 is also available at the CDS via anonymous ftp to cdsarc.u-strasbg. fr (130.79.128.5) or via http://cdsarc. u-strasbg.fr/viz-bin/cat/J/A+A/635/L3
}

is the most prominent stream in the Milky Way halo and has served in multiple studies as a prototype of ongoing tidal disruption and hierarchical formation of the Galaxy, and to constrain the Milky Way potential (Law et al. 2016, and references therein). Nevertheless, reproducing the spatial structure simultaneously with the radial velocities remains a challenge. No single model has succeeded in reproducing all observational data, with the best so far being the triaxial halo by Law \& Majewski (2010a, hereafter LM10) and Deg \& Widrow (2013), which takes the form of a nearly oblate halo but with a minor axis oriented along the Galactic plane. This configuration is infrequent in $\Lambda$ cold dark matter $(\Lambda \mathrm{CDM})$ and is dynamically unstable, but might be avoided by considering the effect of the Large Magellanic Cloud (Vera-Ciro \& Helmi 2013) or a rising Milky Way rotation curve (Ibata et al. 2013). Even so, none of these models can account for other features of the Sgr stream like the bifurcations (Belokurov et al. 2006; Koposov et al. 2012) and substructure near the apocenters (Sesar et al. 2017). However, the modelling efforts (Fellhauer et al. 2006; Peñarrubia et al. 2010; Dierickx \& Loeb 2017) that have managed to reproduce these still suffer from the radial velocity miss-match in the leading arm.

A precise measurement of the proper motion along the Sgr stream could be the observational piece that is missing to solve these long-standing puzzles. So far, determinations of the Sgr proper motion have been very limited: they have 

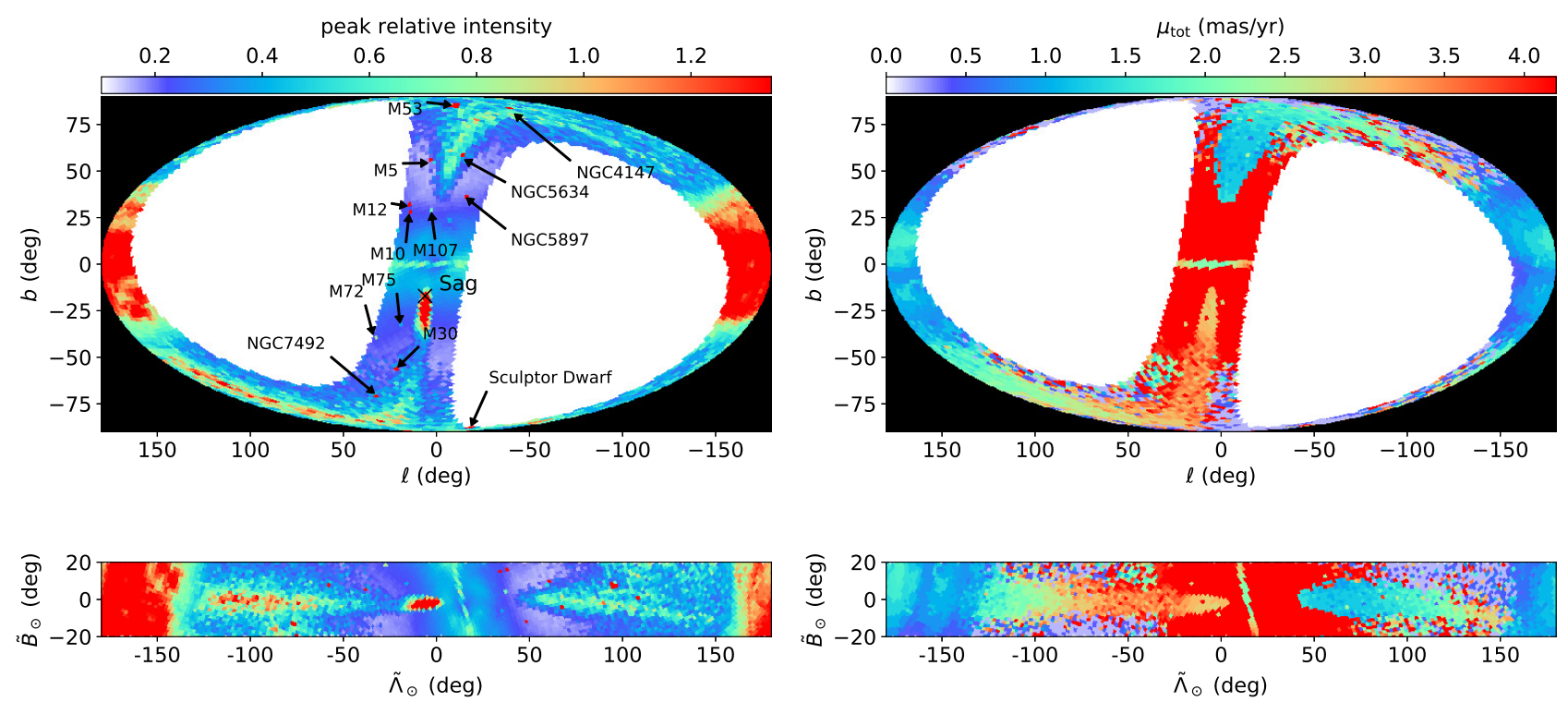

Fig. 1. All-sky view of the Sgr stream with Gaia data in Galactic coordinates (top) and in the coordinates of the orbital plane of Sgr (bottom). Left: HEALpix map in the region of the Sgr stream $\left(\left|\tilde{B}_{\odot}\right| \leq 20 \mathrm{deg}\right)$ colored according to the relative intensity of the most prominent peak detected in the corresponding HEALpix. Known objects such as globular clusters and dwarf galaxies show high relative intensities in their corresponding HEALpix and are marked with arrows. The location of the Sgr dwarf is shown with a black cross; its position was taken from Karachentsev et al. 2004. Right: same as left but colored as a function of the total proper motion $\mu_{\mathrm{tot}}$. In all panels, the trace of the Sgr stream is clearly observed in an almost full circle on the sky, with the leading arm at positive Galactic latitude $b$ (positive $\tilde{\Lambda}_{\odot}$ ) and the trailing arm at negative $b$ (negative $\tilde{\Lambda}_{\odot}$ ).

only been obtained in small patches along the stream and are based on a small number of selected member stars (Carlin et al. 2012; Koposov et al. 2013; Sohn et al. 2015, 2016). Recently, $\mathrm{Li}$ et al. (2019) measured the proper motion of 164 stars from the Large Sky Area Multi-Object Fiber Spectroscopic Telescope (LAMOST) survey (Cui et al. 2012) along the trailing and leading arms with Gaia DR2. Yang et al. (2019) detected substructure with a few hundred members related mostly to the leading arm of the stream in a sample of LAMOST stars with proper motions from Gaia DR2.

Here we determine the proper motion along the Sgr stream using a completely different approach. We use Gaia DR2 astrometry and wavelet transform techniques to conduct a blind detection of over-densities in the proper-motion plane for regions selected broadly in the plane of the stream (Sect. 2). We find, in an unforeseen way, that Sgr accounts for the peak of highest intensity in most of the probed fields (Sect. 3). From this we measure the continuous proper motion along the path of the stream (Sect. 4). By selecting all stars associated to the detected peaks, we explore their Gaia color-magnitude diagram (CMD) and reconstruct the largest sample of candidate members of the Sgr stream (Sect. 5). We discuss the many avenues opened by our findings in Sect. 6.

\section{Data and methods}

We select stars from Gaia DR2 (Gaia Collaboration 2018) with parallax satisfying $\varpi-\sigma_{\varpi}<0.1$ mas and $B P-R P>0.2$. The first cut ensures that we keep stars farther than $10 \mathrm{kpc}$ given their uncertainties and discards most of the foreground. The second cut eliminates blue foreground main sequence stars, while only removing a small fraction of blue horizontal branch stars.

We then look for over-densities in the proper motion covering the whole sky by applying a peak-detection algorithm in the proper-motion plane $\left(\mu_{\alpha_{*}}, \mu_{\delta}\right)$ of each Gaia HEALpix of level 5. The peak detection algorithm is a simplified version of the method presented in Antoja et al. (2015) based on the wavelet transform (WT) and is detailed in Appendix A. In summary, for the proper-motion plane of each HEALpix, the algorithm finds peaks and determines their proper-motion coordinates, their significance, their width or scale, their intensity or height, and the number of stars that belong to the peak. The algorithm detects peaks of a set of discrete widths, which are referred to as scales. Here we explore three logarithmically spaced scales in proper motion of $0.48,0.96$, and $1.92 \mathrm{mas} \mathrm{yr}^{-1}$, that we denote 1,2 , and 3. Finally, we select only the peaks with significance $\geq 3$ and we keep only the ones with the highest relative intensity in each HEALpix, that is, the peak with the largest height in each sky patch.

\section{A Gaia full-sky vision of the Sgr stream}

Figure 1 shows four HEALpix maps of our results. We plot only HEALpix fields with $\left|\tilde{B}_{\odot}\right| \leq 20 \mathrm{deg}$, where $\tilde{B}_{\odot}$ is the latitude from the coordinate system of the $\operatorname{Sgr}$ stream $\left(\tilde{\Lambda}_{\odot}, \tilde{B}_{\odot}\right)$ defined in Majewski et al. (2003) with the orientation adopted in Belokurov et al. (2014a). In the top-left panel, each HEALpix is colored as a function of the relative intensity of the highest peak. This map clearly shows the stream emerging from the Sgr dwarf galaxy. The Sgr dwarf appears as a very strong concentration (red HEALpix) at approximately the center of the map, marked with a black cross. The leading $\operatorname{arm}(b>0)$ is seen in turquoise, while the trailing arm $(b<0)$, which has higher relative intensity, is seen in orange-red. This shows that in most of the sky around the Sgr stream the highest peak detected by our algorithm corresponds to the stream itself. Only where the stream crosses the Galactic plane, i.e., at $\ell \sim 0$ and $\sim 180 \mathrm{deg}$, is another dominant structure apparent, which is probably related to the Milky Way disk. We also see many other HEALpix with prominent peaks that correspond to known objects, mainly globular clusters and dwarf galaxies (some are indicated with black arrows). Their detection corroborates the reliability of our method. 

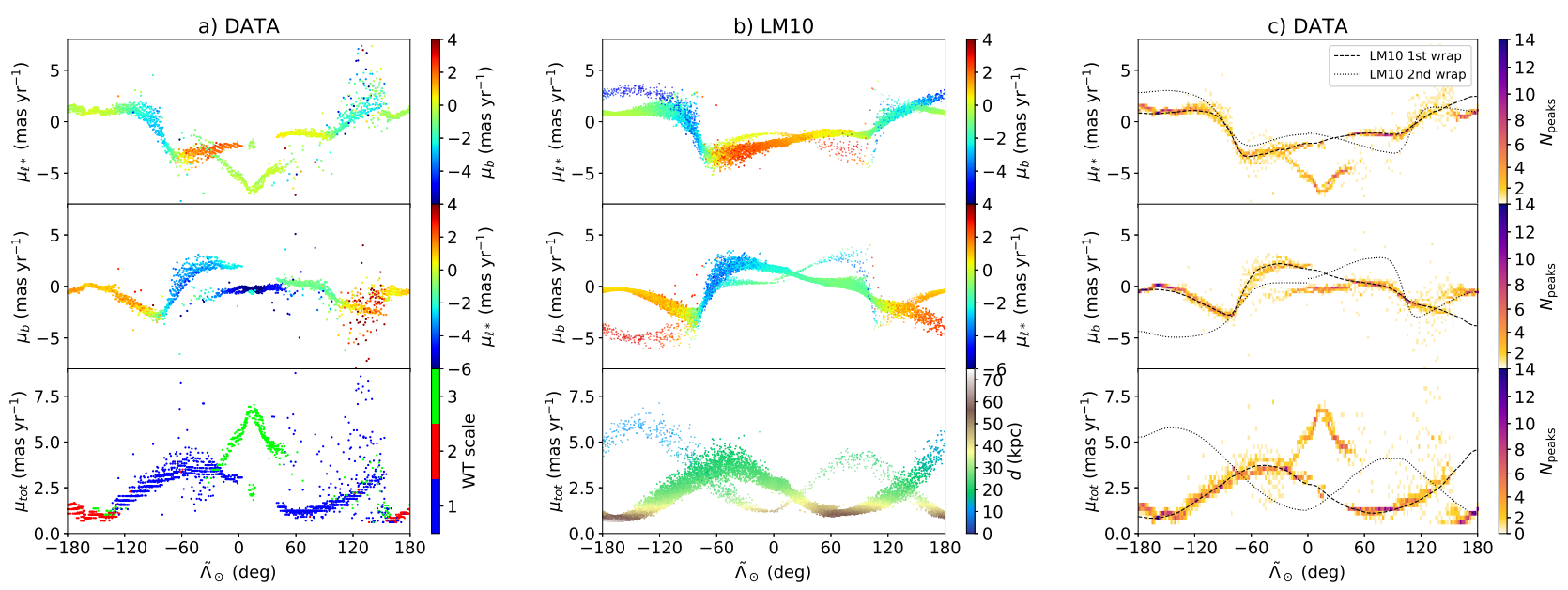

Fig. 2. Proper motion of the Sgr stream from the Gaia data and comparison with the LM10 model. All panels show the proper motion in Galactic longitude in the top, in latitude in the middle, and total in the bottom, as a function of $\tilde{\Lambda}_{\odot}$. (a) Proper motions of the detected peaks color coded by proper motion in latitude (top), in longitude (middle), and in WT scale (bottom). (b) Same but for the model and the bottom panel colored by distance to the Sun. The second wrap of the model has been given more transparency to enhance the contrast of the first wrap. (c) Same as $(a)$ but represented by a $2 \mathrm{D}$ histogram with the trace of the model superposed in black dashed (first wrap) and dotted (second wrap) lines.

The HEALpix map in the top right panel of Fig. 1 is colored by total proper motion $\mu_{\mathrm{tot}}$ of the highest intensity peaks. Again, the Sgr stream appears as a river of HEALpix fields that gradually change their color when moving from the Sgr dwarf towards the leading (blue-green colors) and trailing (orange-green colors) arms. This is the first all-sky view of the Sgr stream in proper motion ever produced.

In the bottom panels, the same HEALpix maps are shown but in the coordinates of the Sgr orbital plane as defined above. A clear bifurcation in the leading arm can be seen in the bottomleft panel from $\tilde{\Lambda}_{\odot} \approx 90 \mathrm{deg}$ to $\approx 140 \mathrm{deg}$, confirming that the discovery by Belokurov et al. (2006) is observed also with Gaia data. However, we do not see the bifurcation in the trailing arm (Koposov et al. 2012), nor do we a signature that the bright and faint parts of the leading arm have different proper motion (bottom right panel).

\section{Proper-motion sequence of the Sgr stream}

The proper motion of the peaks with the highest intensity is shown in Fig. 2 as a function of $\tilde{\Lambda}_{\odot}$, only for $\left|\tilde{B}_{\odot}\right| \leq 10 \mathrm{deg}$ instead of $\left|\tilde{B}_{\odot}\right| \leq 20$ deg as in Fig. 1 in order to be more restrictive. Figure 2a shows the detected peaks in the planes of $\tilde{\Lambda}_{\odot}-\mu_{\ell *}$ (top), $\tilde{\Lambda}_{\odot}-\mu_{b}$ (middle), and $\tilde{\Lambda}_{\odot}-\mu_{\text {tot }}$ (bottom) color coded by $\mu_{b}$ (top), by $\mu_{\ell_{*}}$ (middle), and by the WT scale (bottom). Figure $2 \mathrm{~b}$ is the same as Fig. 2a but for the LM10 model of the Sgr stream, in which we have selected only particles that roughly correspond to the observed parts of the stream ${ }^{1}$. We note that the data panels show the peaks detected in proper-motion space (each one composed of hundreds to thousands of stars), while the panels for the model correspond to particles in the simulation. In the bottom panel of Fig. 2b we color-code them by distance to the Sun. Figure $2 \mathrm{c}$ shows a $2 \mathrm{D}$ histogram of the data peaks. We overplot the LM10 model with black curves obtained by computing the median proper motion in bins of $\tilde{\Lambda}_{\odot}$ and subsequently smoothing with a Gaussian filter. The data show a continuous sequence of proper motion along $\tilde{\Lambda}_{\odot}$ that strikingly resembles that of the LM10 model. We identify the first wrap of the trailing

\footnotetext{
1 Particles in http://faculty.virginia.edu/srm4n/Sgr/ with
} $P_{\text {col }} \leq 3$, i.e. that became unbound recently. and leading arms of the Sgr stream. In addition, a few peaks seem to coincide with the expected position of the trailing arm at $\tilde{\Lambda}_{\odot} \approx 180 \mathrm{deg}$. The continuity of the sequence is only broken where the stream crosses the Galactic disk and is hard to detect.

In the data, there is a set of peaks at $\tilde{\Lambda}_{\odot} \approx[-50,50] \mathrm{deg}$ organized along a triangular shape in the top and bottom panels of Figs. 2a and c that do not belong to Sgr. An analysis of a Gaia mock catalog without the Sgr stream (Fig. B.1) reveals a similar structure. We therefore conclude that this is caused by contamination from the stellar foreground. We filtered out these peaks by removing all peaks of scale 3 (green colors in the bottom panel of Fig. 2a). Additionally, we see peaks that fall outside the sequence around $\tilde{\Lambda}_{\odot} \approx 150 \mathrm{deg}$. These do not appear in the mock catalog but appear in an off-stream sky band selected in the range $15<\left|\tilde{B}_{\odot}\right| \leq 20 \mathrm{deg}$ (Fig. B.2). Their origin at this point is not clear but we note that their location on the sky is similar to that of the Virgo over-density and there is some overlap in proper motion according to the values reported by Yang et al. (2019).

For a more quantitative comparison between the data and the model, we refine the selection of peaks by applying simple geometric filters in the three planes $\left(\tilde{\Lambda}_{\odot}-\mu_{\ell *}, \tilde{\Lambda}_{\odot}-\mu_{\mathrm{b}}, \tilde{\Lambda}_{\odot-\mu_{\mathrm{tot}}}\right)$, intending only to remove peaks that are clearly off the proper-motion sequence. These filters are detailed in Appendix $\mathrm{C}$ and the region where peaks are selected is shown with green lines in Fig. 3a. We finally end up with 1540 peaks which are shown in blue and orange for the trailing and leading arms, respectively. We then retrieve from the Gaia archive the stars associated to each peak by selecting those inside a circle centered on each peak with a radius equal to the corresponding WT scale. From this set of stars we have removed stars from globular clusters as detailed in Appendix D. Our final sample has 2168723 stars.

We then compute the stars median $\mu_{\ell_{*}}, \mu_{\mathrm{b}}$ and $\mu_{\mathrm{tot}}$ in bins of $\tilde{\Lambda}_{\odot}$, which we then smooth with a Gaussian filter, as done previously for the model. We compute the statistical error on the median using the approximation $\sqrt{\frac{\pi}{2}} \frac{\sigma}{\sqrt{N}}$, where $\frac{\sigma}{\sqrt{N}}$ is the standard error on the mean. The curve obtained is shown in Fig. 3a with blue (trailing) and red (leading) solid lines. The error is $<0.03$ mas yr $^{-1}$ for $75 \%$ of the points in the smoothed curves. Again, the black lines correspond to the LM10 model.

Finally, Fig. 3b shows the $\Delta \mu_{\ell_{*}}, \Delta \mu_{\mathrm{b}}$ and $\Delta \mu_{\mathrm{tot}}$ residuals, defined as $\Delta \equiv$ (data) - (model) with the grey area showing 

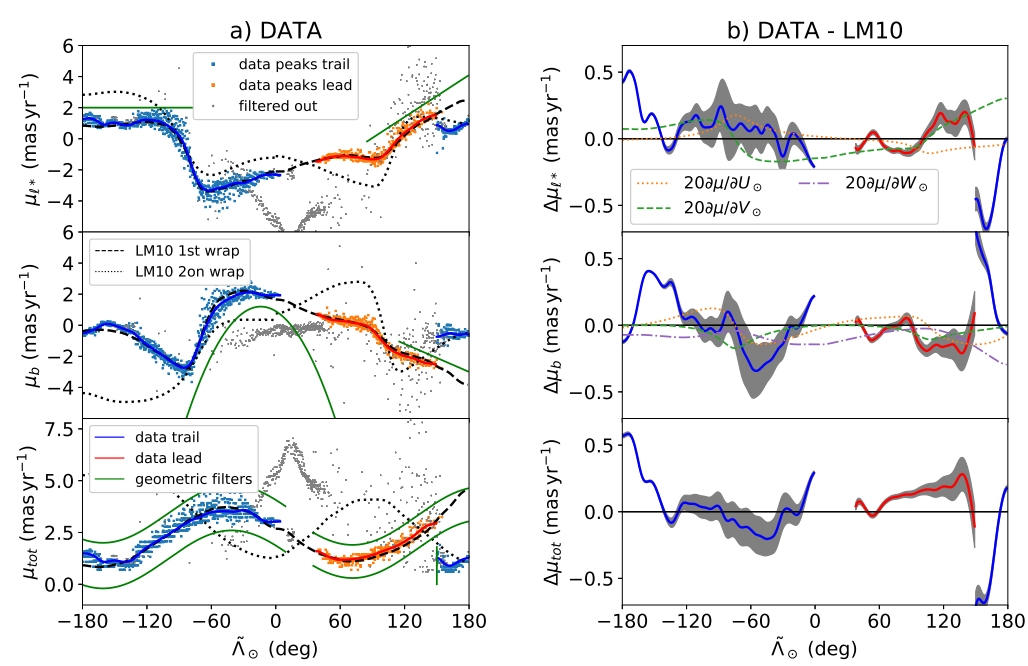

Fig. 3. Proper motion of the Sgr stream from the Gaia data and comparison with the LM10 model (part 2). Top, middle, and bottom panels: organized as in Fig. 2. (a) Proper motions of the peaks finally selected to belong to the Sgr stream colored with blue (trailing) and orange (leading). A smooth version of the median proper motion of the Gaia DR2 stars belonging to the detected peaks (see text) is shown in blue and red, together with the model in black. The green lines indicate the geometric filters that we apply to select the peaks that belong to the main sequences. (b) Differences in the median proper motions between data and model with the shaded areas indicating the statistical error. The rest of the curves indicate the values of a potential discrepancy due to an inappropriate assumption of the solar motion values in LM10 by $20 \mathrm{~km} \mathrm{~s}^{-1}$ (see text).

the combined errors on the median added in quadrature (thus,

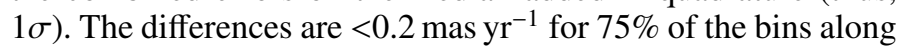
the evaluated sequences. For the leading arm, we see systematic differences $\lesssim 0.2$ mas yr$^{-1}$ in $\mu_{\ell *}$ and $\mu_{\mathrm{b}}$ well above the statistical error and a larger total proper motion than for the model. However, the biggest differences are for the trailing arm at $\tilde{\Lambda}_{\odot}<-120 \mathrm{deg}$ (including its prolongation at $\tilde{\Lambda}_{\odot} \approx 180 \mathrm{deg}$ ). These discrepancies could be due to strong contamination from the foreground (see Appendix B). Indeed, the CMDs analyzed in Sect. 5 appear very contaminated in this range. There are also significant discrepancies near $\tilde{\Lambda}_{\odot} \approx-50 \mathrm{deg}$ in $\mu_{\mathrm{b}}$ that are

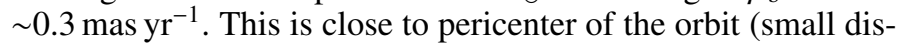
tances; see bottom panel of Fig. 2b). This position is also where the contribution of the solar motion is larger. In Fig. $3 \mathrm{~b}$ we plot the derivative of the contribution of solar motion with respect to the individual components $\left(U_{\odot}, V_{\odot}, W_{\odot}\right)$. A distance needs to be assumed for this calculation, so we take this distance from the LM10 model. We see that the shape of $\frac{\partial \mu_{\ell_{*}}}{\partial U_{\odot}}$ and $\frac{\partial \mu_{\mathrm{b}}}{\partial U_{\odot}}$ is similar to that of the differences between data and model for the trailing arm, but not for the leading arm. However, all the derivatives are very small (at most of $0.01 \mathrm{mas} \mathrm{yr}^{-1} \mathrm{~km} \mathrm{~s}^{-1}$ ). Therefore, the solar motion assumed in the LM10 would have to differ by more than $20 \mathrm{~km} \mathrm{~s}^{-1}$ in order to explain the observed differences. This could be plausible for $V_{\odot}$ (for instance $V_{\odot}=232 \mathrm{~km} \mathrm{~s}^{-1}$ in LM10 but $V_{\odot}=255 \mathrm{~km} \mathrm{~s}^{-1}$ from Reid et al. 2014) and therefore at this point we cannot discard the possibility that a combination of a different $V_{\odot}$ together with a distance difference is the cause of part (but not all) of the proper-motion discrepancies.

The systematic errors in the Gaia proper motions cannot account for the observed residuals since they are much smaller (of the order of $0.028 \mathrm{mas} \mathrm{yr}^{-1}$ at the scales of $\sim 14-20 \mathrm{deg}$; see Table 4 in Lindegren et al. 2018).

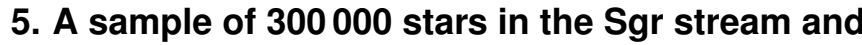 dwarf}

In Fig. 4 we plot the CMDs of the stars that belong to the detected peaks of Sgr in bins of $15 \mathrm{deg}$ in $\tilde{\Lambda}_{\odot}$. We have organized the panels so that the detection of the trailing arm at $\tilde{\Lambda}_{\odot}=$ $180 \mathrm{deg}$ is connected to that at $\tilde{\Lambda}_{\odot}=-180 \mathrm{deg}$. In the direction of the Galactic center, there were no selected peaks, hence the blank panel. Except for the panels of the first row that are close to the Galactic plane in the anti-center direction (see discussion below), the signature of Sgr is clear, with prominent red clump (RC), red giant branch, extended horizontal branch, and turn-off stars. The Sgr dwarf itself has a high density of stars in the range $\tilde{\Lambda}_{\odot}=[-15,15] \mathrm{deg}$ (second and third panels of the third row).

In Fig. 4 we observe the position of the RC moving continuously in $G$ magnitude as a function of $\tilde{\Lambda}_{\odot}$ due to the varying distance of the stream. This is better seen in Fig. 5 where we show the apparent distance modulus versus $\tilde{\Lambda}_{\odot}$, computed as if all stars were RC stars, assuming a RC absolute magnitude of $M_{\mathrm{G}}=0.495$ (Ruiz-Dern et al. 2018) and neglecting effects of extinction and of the metallicity gradient along the stream (e.g., Keller et al. 2010; Hayes et al. 2020). The distance trend shows general good agreement with the LM10 model (blue curves, right axis). The leading arm appears to be $\sim 5 \mathrm{kpc}$ closer than predicted by the model at $\tilde{\Lambda}_{\odot} \approx 100 \mathrm{deg}$, which cannot be caused by extinction since this would produce the contrary effect, and we see a shift in the angular position of the apocenter at around $\tilde{\Lambda}_{\odot} \approx 70 \mathrm{deg}$. A more thorough comparison of the distances from the data and model is the subject of another work (Ramos et al., in prep).

We note that an incorrect distance in the LM10 model would also cause a discrepancy in the proper motions. For instance, an overestimation of the model distance for the leading arm by $10 \%$ will cause an overall underestimation of the proper motion by $10 \%$, which is similar to what we observe.

It is hard to identify the Sgr trailing stream in the CMDs of the first row of Fig. 4 where there is strong contamination. The RC of the trailing arm should be quite faint here given the distances of this part of the stream $(\sim 50 \mathrm{kpc}$ according to LM10) and is probably hidden inside the big blob of contaminating stars. We only see the emerging tip of the giant branch at $\tilde{\Lambda}_{\odot}=[150,180] \mathrm{deg}$ (plume of stars at $G_{\mathrm{BP}}-G_{\mathrm{RP}} \approx 1.75$ ) and some hints of it at $\tilde{\Lambda}_{\odot}=[-150,-120] \mathrm{deg}$. It is very 


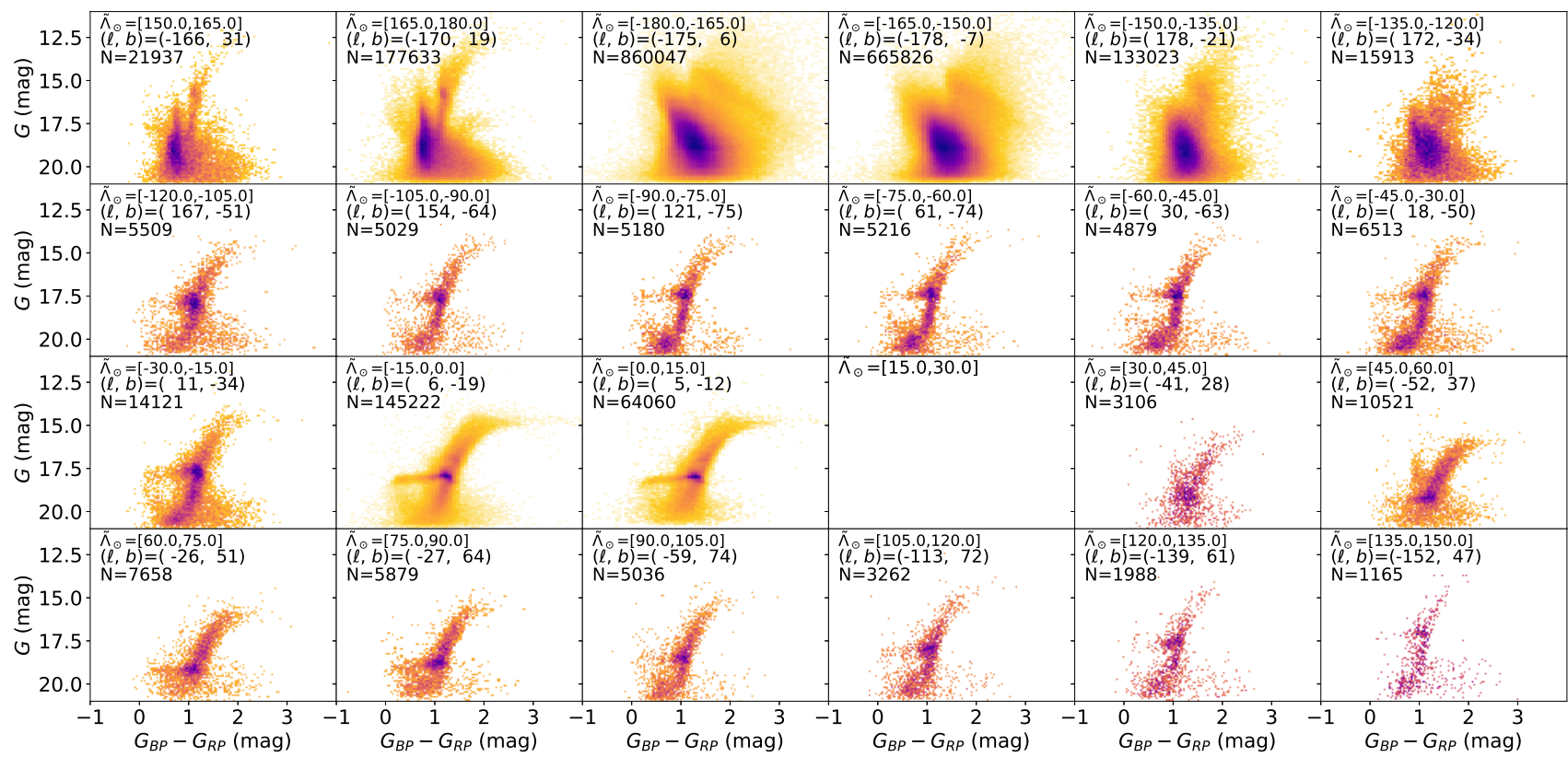

Fig. 4. Color-magnitude diagrams of the stars belonging to the proper motion peaks classified as Sgr peaks for different bins in $\tilde{\Lambda}_{\odot}$ as indicated in the legend. The corresponding means in Galactic coordinates of the stars are also shown. The CMDs at higher Galactic latitudes are dominated by the Sgr population.

unlikely that the RC that we see in the first five panels of Fig. 4 belongs to the leading arm in its second wrap: their distance inferred from their magnitude could be compatible with this second wrap but not their proper motion ${ }^{2}$. The structure seen in these panels could alternatively be due to contamination of the disk or disks, the flare, the warp, or other outer disk substructures (Xu et al. 2015) such as the Monoceros ring (Newberg et al. 2002) or TriAnd overdensity (Rocha-Pinto et al. 2004; Sheffield et al. 2014; Price-Whelan et al. 2015).

We note that our selection of member stars is contaminated in certain areas and that the completeness resulting from our methodology is yet to be determined. While we plan to deal with these in the future, here we build a list of member candidates by selecting stars in the ranges of $\tilde{\Lambda}_{\odot} \in[-120,-10]$ deg (trailing arm) and of $\tilde{\Lambda}_{\odot} \in[30,150]$ deg (leading arm) where the contamination is minimal. We count 61937 and 38615 stars, in the trailing and leading arms, respectively, making a total of 100552 stars of different stellar types along the stream of Sgr. We also find 193792 stars in the approximate region of the Sgr dwarf $\left(\tilde{\Lambda}_{\odot}=[-10,10] \mathrm{deg}\right)$, given an apparent size of $15 \mathrm{deg}$ (e.g., Giuffrida et al. 2010).

The list of member candidates (see Table E.1), the median proper motions of the data, the interpolation used to obtain the smooth curves given as a pickle Python object, and additional material will be made publicly available online ${ }^{3}$ and through CDS.

\section{Discussion and conclusions}

Here, we present a measurement of the proper motion of the Sgr stream with Gaia DR2 data. Compared to previous studies based on a small number of stars located in a few particular

\footnotetext{
2 Indeed, the proper-motion difference of the two branches is about 5 mas $\mathrm{yr}^{-1}$ according to the LM10 model, which is much larger than the expected dispersion of the stream considering the intrinsic dispersion and also the dispersion produced by errors.

3 https://services.fqa.ub.edu/sagittarius
}

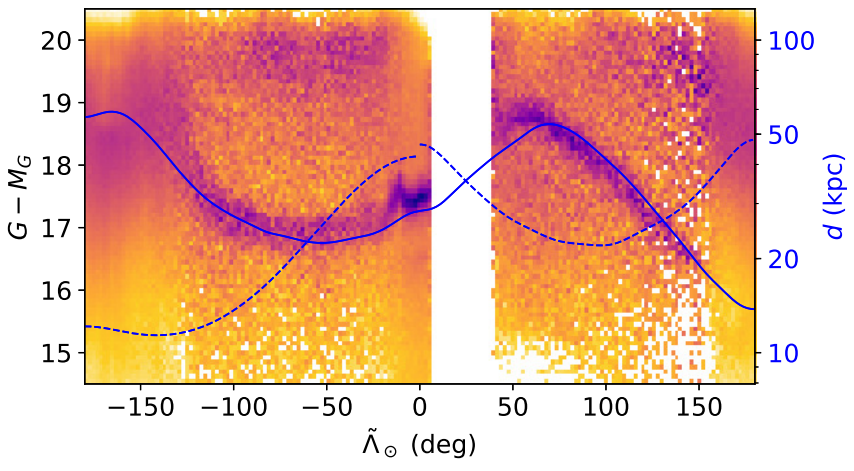

Fig. 5. Apparent distance modulus as a function of $\tilde{\Lambda}_{\odot}$ for the stars belonging to the Sgr stream and Sgr dwarf. The data are shown as a histogram normalized by column. The stars belonging to the RC depict a well-defined sequence. In a secondary axis, the distance of the particles in the LM10 model is superposed in blue. In the absence of extinction and with our assumed calibration of the $\mathrm{RC}$, the two would be directly comparable. The data show general good agreement with the model with discrepancies for example in the apocenter of the leading arm.

fields (Carlin et al. 2013; Koposov et al. 2013; Sohn et al. 2015, 2016), our determination is substantially better because: i) it is based on a much greater number of stars (around 100000 stars in the stream) and covers different stellar types; and ii) is, for the first time, a continuous determination that extends, except for the regions behind the Galactic plane, $2 \pi$ in the sky. This makes it the proper motion determination with the largest span for any stream to date. We also clearly detect the bifurcation of the leading arm. The whole sky coverage and precision of the proper motions given by Gaia are key to the success of the present study.

Our determination comes directly from the detection of peaks of stars in the proper-motion plane covering the sky continuously. That is, we do not use a previous selection of Sgr 
members, but the member candidates are rather a by-product of our study. In this sense, it is the first time that the stream is detected based on a proper-motion search. Moreover, our search is a blind one, meaning that the peaks corresponding to the overdensity of the stream stand out naturally as the peaks with highest intensity in the proper-motion distribution of each sky patch. We note that such a continuous determination would be hard to achieve using match filtering techniques since the distance to the stream changes across the sky. Moreover, our method does not require any assumptions, contrary to other methods that also use proper-motion information for the stream detection, such as for example in STREAMFINDER (Malhan et al. 2018a), where the authors look for trajectories coherent with a stream shape in a given gravitational potential. In fact, in the final stages of this work we found out that a simultaneous study by Ibata et al. (priv. comm.) has also detected the Sgr stream with Gaia data using this completely different methodology and it will be extremely interesting to compare their results with ours in the near future. However, we note that our ability to detect new streams, as done with STREAMFINDER, has still to be confirmed.

We note that we detected several globular clusters along the path of the Sgr stream that also fall in the same propermotion sequence as Sgr. From these, there is consensus about the likely relation of Pal 12, Arp 2, M 54, Ter 7, and Ter 8 with Sgr (e.g., Law \& Majewski 2010b; Massari et al. 2019), and more recently of NGC 2419 (Belokurov et al. 2014b; Massari et al. 2017; Sohn et al. 2018) that we confirm here, while not for NGC 5053 and NGC 5024 (Sohn et al. 2018; Tang et al. 2018).

Our list of member candidates contains about 100000 stars in the stream and 200000 stars in the Sgr dwarf itself and opens up many possibilities for further investigations, such as on the population of stars along the stream, the distance to the stream using RC stars, the width of the stream in the sky and its velocity dispersion, the determination of the solar motion (Hayes et al. 2018), and the possible detection gaps and spurs which are expected (Amorisco 2015) and seen in other streams (Price-Whelan \& Bonaca 2018; Erkal et al. 2019). The continuity and span in the sky of our proper-motion measurement allows for determination of the 3D kinematics of the Sgr stream along its first $2 \pi \mathrm{deg}$, which could hold valuable information for an improvement of the models of the gravitational potential of the Galaxy and companions such as the LMC influencing the Sgr orbit (Vera-Ciro \& Helmi 2013).

Acknowledgements. We thank the anonymous referee for his/her helpful comments. This work has made use of data from the European Space Agency (ESA) mission Gaia (https://www . cosmos. esa.int/gaia), processed by the Gaia Data Processing and Analysis Consortium (DPAC, https://www.cosmos. esa.int/web/gaia/dpac/consortium). Funding for the DPAC has been provided by national institutions, in particular the institutions participating in the Gaia Multilateral Agreement. This project has received funding from the European Union's Horizon 2020 research and innovation programme under the Marie Skłodowska-Curie grant agreement No. 745617 and No. 800502. This work was supported by the MINECO (Spanish Ministry of Economy) through gran ESP2016-80079-C2-1-R and RTI2018-095076-B-C21 (MINECO/FEDER, UE), and MDM-2014-0369 of ICCUB (Unidad de Excelencia "María de Maeztu"). This project has received support from the DGAPA/UNAM PAPIIT program grant IG100319. AH acknowledges financial support from a VICI grant from the Netherlands Organisation for Scientific Research, NWO. JAC-B acknowledges financial support to CAS-CONICYT 17003. CM is grateful for the hospitality of the ICCUB, during visits in which part of this research was carried out.

\section{References}

Amorisco, N. C. 2015, MNRAS, 450, 575

Antoja, T., Mateu, C., Aguilar, L., et al. 2015, MNRAS, 453, 541

Baumgardt, H., \& Hilker, M. 2018, MNRAS, 478, 1520
Belokurov, V., Zucker, D. B., Evans, N. W., et al. 2006, ApJ, 642, L137 Belokurov, V., Irwin, M. J., Koposov, S. E., et al. 2014a, MNRAS, 441, 2124 Belokurov, V., Koposov, S. E., Evans, N. W., et al. 2014b, MNRAS, 437, 116 Bonaca, A., Hogg, D. W., Price-Whelan, A. M., \& Conroy, C. 2019a, ApJ, 880, 38

Bonaca, A., Conroy, C., Price-Whelan, A. M., \& Hogg, D. W. 2019b, ApJ, 881, L37

Carlin, J. L., Majewski, S. R., Casetti-Dinescu, D. I., et al. 2012, ApJ, 744, 25 Carlin, J. L., DeLaunay, J., Newberg, H. J., et al. 2013, ApJ, 777, L5 Cui, X.-Q., Zhao, Y.-H., Chu, Y.-Q., et al. 2012, Res. Astron. Astrophys., 12, 1197

Deg, N., \& Widrow, L. 2013, MNRAS, 428, 912

Dierickx, M. I. P., \& Loeb, A. 2017, ApJ, 836, 92

Erkal, D., Belokurov, V., Laporte, C. F. P., et al. 2019, MNRAS, 487, 2685

Fellhauer, M., Belokurov, V., Evans, N. W., et al. 2006, ApJ, 651, 167

Gaia Collaboration (Prusti, T., et al.) 2016, A\&A, 595, A1

Gaia Collaboration (Brown, A. G. A., et al.) 2018, A\&A, 616, A1

Giuffrida, G., Sbordone, L., Zaggia, S., et al. 2010, A\&A, 513, A62

Hayes, C. R., Law, D. R., \& Majewski, S. R. 2018, ApJ, 867, L20

Hayes, C. R., Majewski, S. R., Hasselquist, S., et al. 2020, ApJ, 889, 63

Ibata, R. A., Gilmore, G., \& Irwin, M. J. 1994, Nature, 370, 194

Ibata, R., Lewis, G. F., Martin, N. F., Bellazzini, M., \& Correnti, M. 2013, ApJ, 765, L15

Ibata, R. A., Malhan, K., Martin, N. F., \& Starkenburg, E. 2018, ApJ, 865, 85

Ibata, R. A., Bellazzini, M., Malhan, K., Martin, N., \& Bianchini, P. 2019a, Nat. Astron., 3, 667

Ibata, R. A., Malhan, K., \& Martin, N. F. 2019b, ApJ, 872, 152

Karachentsev, I. D., Karachentseva, V. E., Huchtmeier, W. K., \& Makarov, D. I. 2004, AJ, 127, 2031

Keller, S. C., Yong, D., \& Da Costa, G. S. 2010, ApJ, 720, 940

Koposov, S. E., Belokurov, V., Evans, N. W., et al. 2012, ApJ, 750, 80

Koposov, S. E., Belokurov, V., \& Evans, N. W. 2013, ApJ, 766, 79

Koposov, S. E., Belokurov, V., Li, T. S., et al. 2019, MNRAS, 485, 4726

Law, D. R., \& Majewski, S. R. 2010a, ApJ, 714, 229

Law, D. R., \& Majewski, S. R. 2010b, ApJ, 718, 1128

Law, D. R., \& Majewski, S. R. 2016, in Tidal Streams in the Local Group and Beyond, eds. H. J. Newberg, \& J. L. Carlin, Astrophys. Space Sci. Lib., 420, 31

Li, J., Fellow, L., Liu, C., et al. 2019, ApJ, 874, 138

Lindegren, L., Hernández, J., Bombrun, A., et al. 2018, A\&A, 616, A2

Majewski, S. R., Skrutskie, M. F., Weinberg, M. D., \& Ostheimer, J. C. 2003, ApJ, 599, 1082

Malhan, K., Ibata, R. A., \& Martin, N. F. 2018a, MNRAS, 481, 3442

Malhan, K., Ibata, R. A., Goldman, B., et al. 2018b, MNRAS, 478, 3862

Malhan, K., Ibata, R. A., Carlberg, R. G., Valluri, M., \& Freese, K. 2019, ApJ, 881, 106

Massari, D., Posti, L., Helmi, A., Fiorentino, G., \& Tolstoy, E. 2017, A\&A, 598, L9

Massari, D., Koppelman, H. H., \& Helmi, A. 2019, A\&A, 630, L4

Mateo, M., Mirabal, N., Udalski, A., et al. 1996, ApJ, 458, L13

Newberg, H. J., Yanny, B., Rockosi, C., et al. 2002, ApJ, 569, 245

Palau, C. G., \& Miralda-Escudé, J. 2019, MNRAS, 488, 1535

Peñarrubia, J., Belokurov, V., Evans, N. W., et al. 2010, MNRAS, 408, L26

Price-Whelan, A. M., \& Bonaca, A. 2018, ApJ, 863, L20

Price-Whelan, A. M., Johnston, K. V., Sheffield, A. A., Laporte, C. F. P., \& Sesar, B. 2015, MNRAS, 452, 676

Ramos, P., Antoja, T., \& Figueras, F. 2018, A\&A, 619, A72

Reid, M. J., Menten, K. M., Brunthaler, A., et al. 2014, ApJ, 783, 130

Rocha-Pinto, H. J., Majewski, S. R., Skrutskie, M. F., Crane, J. D., \& Patterson,

R. J. 2004, ApJ, 615, 732

Ruiz-Dern, L., Babusiaux, C., Arenou, F., Turon, C., \& Lallement, R. 2018, A\&A, 609, A116

Rybizki, J., Demleitner, M., Fouesneau, M., et al. 2018, PASP, 130, 074101

Sesar, B., Hernitschek, N., Dierickx, M. I. P., Fardal, M. A., \& Rix, H.-W. 2017, ApJ, 844, L4

Sheffield, A. A., Johnston, K. V., Majewski, S. R., et al. 2014, ApJ, 793, 62

Shipp, N., Li, T. S., Pace, A. B., et al. 2019, ApJ, 885, 3

Sohn, S. T., van der Marel, R. P., Carlin, J. L., et al. 2015, ApJ, 803, 56

Sohn, S. T., van der Marel, R. P., Kallivayalil, N., et al. 2016, ApJ, 833, 235

Sohn, S. T., Watkins, L. L., Fardal, M. A., et al. 2018, ApJ, 862, 52

Starck, J.-L., \& Murtagh, F. 2002, in Astronomical Image and Data Analysis, eds. J.-L. Starck, \& F. Murtagh (Springer)

Tang, B., Fernández-Trincado, J. G., Geisler, D., et al. 2018, ApJ, 855, 38

Totten, E. J., \& Irwin, M. J. 1998, MNRAS, 294, 1

van der Walt, S., Schönberger, J. L., Nunez-Iglesias, J., et al. 2014, PeerJ, 2, e453

Vera-Ciro, C., \& Helmi, A. 2013, ApJ, 773, L4

Xu, Y., Newberg, H. J., Carlin, J. L., et al. 2015, ApJ, 801, 105

Yang, C., Xue, X.-X., Li, J., et al. 2019, ApJ, 880, 65 


\section{Appendix A: Peak-detection algorithm and query in the Gaia archive}

The peak detection algorithm is a simplified version of the method presented in Antoja et al. (2015). Here we summarize the algorithm, but more technical details and recent applications can be found in Starck \& Murtagh (2002), Antoja et al. (2015), and Ramos et al. (2018).

The main piece of the algorithm is the a trous ("with holes") variant of the wavelet transform (WT, Starck \& Murtagh 2002), which computes a discrete set of scale-related "views" of a 2D image, where substructure at a particular scale is highlighted. We use the implementation in the Multi Resolution software ${ }^{4}$. For each HEALpix, our initial image is the 2D histogram in

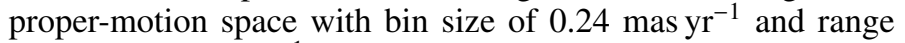

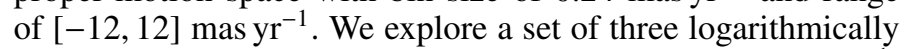

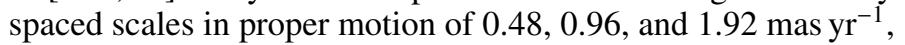
which we denote 1,2 , and 3. An example of the WT application is presented in Fig. A.1, where the WT coefficients are shown in a blue (red) color scale corresponding to over-dense (underdense) regions, for the three different scales (columns) and for two different sky positions as explained in the legends (rows).

Then we search for local peaks in the WT plane at each scale, using peak_local_max from the Python package Scikit-image (van der Walt et al. 2014). The WT also gives the significance of the coefficients based on a model for the Poisson noise. From all the peaks detected, we select only those with WT coefficient with significance ${ }^{5} \geq 3$, which corresponds to $\sigma \geq 3$ in the Gaussian-equivalent case. The peaks detected in each scale are marked with crosses in Fig. A.1. In these plots, which correspond to a HEALpix centered close to the Sgr dwarf (top) and the leading arm (bottom), respectively, a prominent peak is found (green cross) that corresponds to Sgr, as we discuss in the main text.

Finally, the method yields the list of significant peaks in proper motion at each HEALpix with their associated quantities such as the location of the peak in $\mu_{\alpha *}$ and $\mu_{\delta}$, the wavelet transform coefficient WT, which can be thought of as the intensity of the peak, the scale where it was detected (1,2 or 3$)$, and the number of stars belonging to the peak (computed as described in Ramos et al. 2018). A measure of the relative intensity of a peak can be computed as:

$\frac{W T}{N_{\mathrm{hp}}} \times 1000$

where $N_{\mathrm{hp}}$ is the total number of stars in the HEALpix and the 1000 factor is used to convert to more usable numbers (typical relative peak intensities will be of the order of 1).

The peak associated to the Sgr stream is often detected at more than one scale in each HEALpix as exemplified in Fig. A.1. However, the peak will have highest relative intensity at the scale that is closer to its actual size (typically, as in Fig. A.1, this occurs at scale 1 , that is 0.48 mas $\mathrm{yr}^{-1}$ ). We select only the peaks with the highest relative intensity for each HEALpix, which naturally removes redundant peaks.

Due to the impracticality of downloading all the stars that fulfill the cuts imposed, we process in parallel each HEALpix downloading from the Gaia Archive directly the proper-motion histograms using:

SELECT COUNT (*) as N, pmra_index*binsize as pmra, pmdec_index*binsize as pmdec FROM

\footnotetext{
4 http://www.multiresolutions.com/mr/

5 This is equivalent to $W P \geq 99.7$ in the notation of Antoja et al. (2015) and to $C L=3$ in Ramos et al. (2018).
}

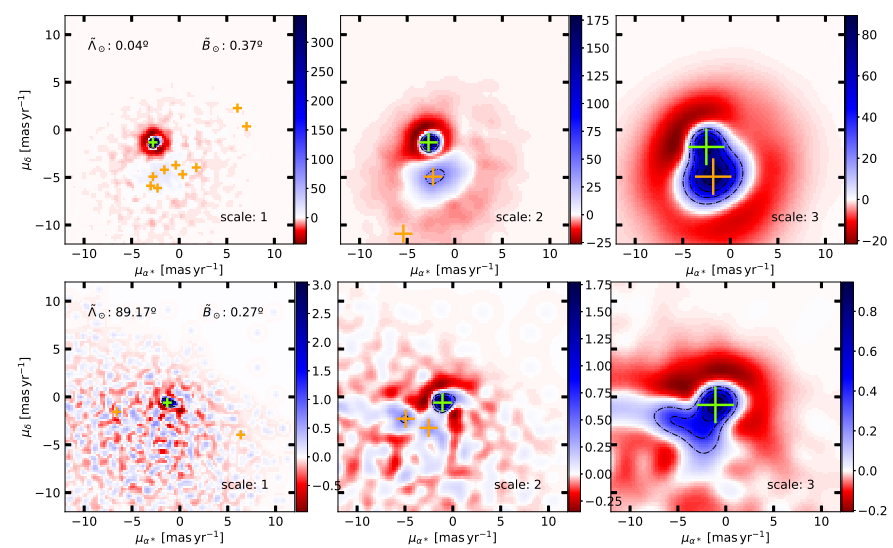

Fig. A.1. Example of WT planes in two different HEALpix fields corresponding to the Sgr core (top row) and the middle part of the leading arm (bottom) at the three different scales analyzed here (three columns, from left to right for small to large). The color indicates the value of the WT coefficients, where darker blue means higher WT, i.e., higher intensity of the substructure, while red colors are for under-dense regions. Crosses are peaks detected in these WT planes. The peak of the Sgr stream/dwarf is marked in green and appears to be well separated from the general field.

\section{(SELECT source_id, FLOOR(pmra/binsize)}

AS pmra_index, FLOOR (pmdec/binsize) AS

pmdec_index FROM gaiadr2.gaia_source WHERE

source_id BETWEEN hpnum*2**35*4**(12-level)

AND (hpnum +1$) * 2 * * 35 * 4 * *(12-$ level) AND

parallax-parallax_error $<0.1$ AND bp_rp $>=0.2$

AND pmra IS NOT Null AND pmdec IS NOT NULL) as

sub GROUP BY pmra_index, pmdec_index

where one should replace hpnum, level and binsize by the number of the HEALpix, the level of the HEALpix grid (here 5) used,

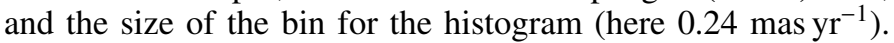
This results in fast queries that return easy to handle small size files. We then apply the WT and peak search algorithm. With a regular computer and eight CPU cores running, the whole sky execution takes on the order of $12 \mathrm{~h}$.

In several HEALpix, we found peaks at $\left(\mu_{\alpha^{*}}, \mu_{\delta}\right) \approx(0,0)$. These are triggered by quasars: in several regions of the sky, especially where the Gaia coverage has been larger (e.g., large visibility_periods_used), quasars are observed more easily and appear as concentrations of sources with almost zero proper motion. Those peaks have been removed by selecting only peaks with $\mu_{\mathrm{tot}} \equiv \sqrt{\mu_{\alpha *}^{2}+\mu_{\delta}^{2}}>0.6 \mathrm{mas} \mathrm{yr}^{-1}$. Although this could be hindering the detection of the parts of the stream with small proper motions, most of these occur in areas that appear to be strongly contaminated by the foreground and would not have entered our selection (see Sect. 4). We also note that the LM10

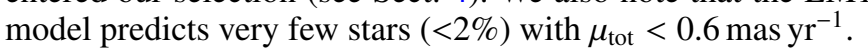

\section{Appendix B: Tests with mock Gaia DR2 data and fields off-stream}

Figure B.1 shows the peaks detected in a mock sample that does not contain the Sgr stream. To built this simulated catalog we took the mock data from Rybizki et al. (2018) and added Gaussian errors to color, magnitude, parallax, and proper motions $\left(\mu_{\alpha^{*}}, \mu_{\delta}\right)$ with the uncertainties quoted. We select stars in the same manner as for the real DR2 data and we apply the same method to detect peaks in proper motion. 

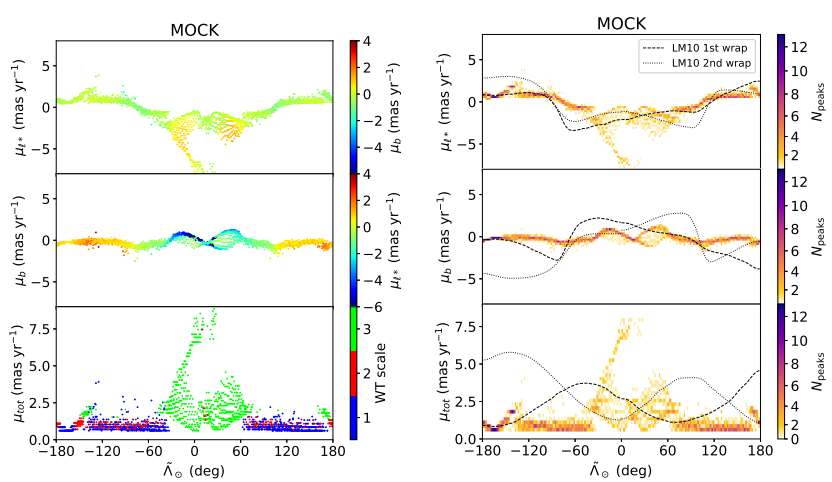

Fig. B.1. Peaks detected in the mock Gaia catalog with no Sgr stream. Left: proper motions of the peaks as a function of $\tilde{\Lambda}_{\odot}$ (top: proper motion in Galactic longitude, middle: in latitude, bottom: total proper motion) color coded by proper motion in latitude (top), in longitude (middle), and by WT scale (bottom). Right: same as in the left panel but in a 2D histogram with the LM10 model superposed with black lines.
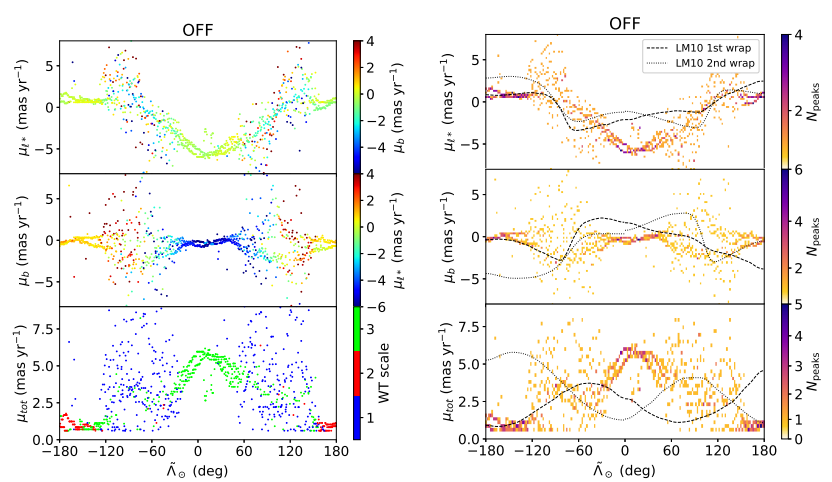

Fig. B.2. Same as Fig. B.1 but for the fields off-stream in the Gaia DR2 data.

The mock data presents a sequence of peaks evolving with $\tilde{\Lambda}_{\odot}$ which most likely correspond to the center of the main component/s of the distribution (mostly foreground stars) that changes with sky position due to differences in the true mean motion of the stars and to the projection in equatorial proper motions of the solar motion. In general, we see that the sequence in the mock catalog and in the data do not match, confirming that the peaks that we assign to Sgr do not belong to the distribution of foreground stars. The exception is the group of peaks organized in a triangular shape at middle $\tilde{\Lambda}_{\odot}$, which as explained in the main text, have been removed for our analysis.

We also note that around $\tilde{\Lambda}_{\odot} \approx-180 \mathrm{deg}$ there are mock peaks that are very close to the LM10 model in all three components $\left(\mu_{\ell_{*}}, \mu_{\mathrm{b}}, \mu_{\mathrm{tot}}\right)$. This is unfortunate since in this position the stream crosses the Galactic plane and therefore we expect the contamination to be very important. This means that the peak corresponding to the stream will be hardly distinguishable from the one for the foreground population. At $\tilde{\Lambda}_{\odot} \approx 180 \mathrm{deg}$ the mock data also show coincidence with the model for the first wrap but only for $\mu_{\ell_{*}}$, which means that the stream could be distinguished unambiguously from the other component $\mu_{\mathrm{b}}$. However, the second wrap of the trailing arm does have a proper motion similar to that of the field.

We have also run our method on a sample of stars off-stream defined as stars with $15<\left|\tilde{B}_{\odot}\right| \leq 20 \mathrm{deg}$. The corresponding plot of peaks is shown in Fig. B.2. We note again the coincidence of peaks that are not Sgr but have the same proper motion at $\tilde{\Lambda}_{\odot} \pm 180 \mathrm{deg}$, further evidencing the contamination when the stream crosses the Galactic plane. We note again the central triangular distribution of peaks that clearly do not belong to Sgr.

\section{Appendix C: Selection of the Sagittarius peaks}

The selection of peaks of the different parts of the stream is done following the following empirical prescriptions. We first define the following lines, where proper motions are given in mas $\mathrm{yr}^{-1}$ :

$$
\begin{aligned}
& \mu_{\text {tot } 1}=2.3-1.4 \sin \left(1.5 \tilde{\Lambda}_{\odot}(\mathrm{rad})-0.5\right) \\
& \mu_{\text {tot } 2}=2.5-1.4 \sin \left(1.5 \tilde{\Lambda}_{\odot}(\mathrm{rad})-0.3\right) \\
& \mu_{\ell_{* 3}}=2.4 \\
& \mu_{\ell * 4}=-4 .+0.045 \tilde{\Lambda}_{\odot} \\
& \mu_{\mathrm{b} 5}=1.2-(1 . / 4 . \times 0.006)\left(\tilde{\Lambda}_{\odot}+14 .\right)^{2} \\
& \mu_{\ell * 6}=2.4-0.03 \tilde{\Lambda}_{\odot} .
\end{aligned}
$$

We then select only peaks satisfying the following criteria for the first part of the trailing $\operatorname{arm}\left(\tilde{\Lambda}_{\odot}<0 \mathrm{deg}\right)$ :

$$
\begin{aligned}
& \left|\mu_{\mathrm{tot}}-\mu_{\mathrm{tot} 1}\right|<1.1 \\
& \mu_{\ell *}<\mu_{\ell * 3} \\
& \mu_{\mathrm{b}}<\mu_{\mathrm{b} 5},
\end{aligned}
$$

for the trailing small part at $\tilde{\Lambda}_{\odot}>150 \mathrm{deg}$ :

$\left|\mu_{\text {tot }}-\mu_{\text {tot } 2}\right|<-1$,

and for the leading part $\left(\tilde{\Lambda}_{\odot}>0\right.$ deg $)$ :

$$
\begin{aligned}
& \left|\mu_{\mathrm{tot}}-\mu_{\mathrm{tot} 2}\right|<0.7 \\
& \mu_{\ell *}<\mu_{\ell_{*}} \text { for } \tilde{\Lambda}_{\odot}<85 \mathrm{deg} \\
& \mu_{\mathrm{b}}<\mu_{\mathrm{b} 6} \text { for } \tilde{\Lambda}_{\odot}>115 \mathrm{deg} .
\end{aligned}
$$

After applying the above geometric filters and examining the proper motion in equatorial coordinates of the selected peaks, there is a peak that is clearly off the Sgr track in $\mu_{\alpha *}$ (while our selection is done in $\mu_{\ell_{*}}-\mu_{\mathrm{b}}$ ). This is caused by an abrupt change of proper motion on certain parts of the celestial sphere related to the coordinate transformation. Thus, we further remove this peak (HEALpix 4491, ICRS level 5).

\section{Appendix D: Removing stars from globular clusters}

From the peaks of high relative intensities, the ones for the globular clusters Pal 12, Terzan 7, Terzan 8, Arp 2, M 54, NGC 2419, M 53 (NGC 5024) and NGC 5053 coincide with the projected path of the Sgr stream and, more importantly, with the propermotion sequence shown in the Fig. 3. We remove the mentioned globular clusters in Fig. 4 and from the list of candidate members by filtering out stars in these clusters. For this we consider the positions, tidal radius and distance from Baumgardt \& Hilker (2018), and take the apparent size of the clusters as 5 times the tidal radius, except for NGC5024 and NGC6715 for which we use factors of 10 and 25 , respectively. 


\section{Appendix E: Candidate members table}

Table E.1 (available at the CDS) contains the data of the candidate members of the Sgr stream and dwarf delivered in this study in the range $-120 \mathrm{deg}<\tilde{\Lambda}_{\odot}<150 \mathrm{deg}$, i.e. avoiding the region of the Galactic plane. We note that these members have been selected based on proper motion only and that a certain fraction of contamination is expected. Here we show an excerpt from the table with the first five rows.

Table E.1. Candidate members of the Sgr stream and dwarf (precision has been reduced here).

\begin{tabular}{ccccccc}
\hline \hline Gaia Source_id & $\alpha$ & $\delta$ & $\mu_{\alpha *}$ & $\mu_{\delta}$ & $G$ & $G_{\mathrm{BP}}-G_{\mathrm{RP}}$ \\
\hline 11922829223296 & 45.2822276579 & 0.3093182817 & 0.5029273104 & -1.6911386321 & 18.21442 & 1.3065739 \\
13400298143232 & 45.0983817638 & 0.2573337519 & 0.5391000357 & -1.7131457615 & 17.18689 & 0.8256264 \\
16870632320128 & 45.1122641436 & 0.3793731413 & -0.0038042018 & -1.8952268886 & 16.70960 & 0.9880648 \\
22024592364160 & 44.7397533562 & 0.2424985998 & -0.0589970570 & -1.9336723389 & 18.68879 & 1.0945892 \\
46660524732544 & 45.6057094408 & 0.5823213123 & 0.3125358412 & -1.5970907824 & 18.31924 & 1.2143192 \\
\hline
\end{tabular}

Notes. The full table is available at the CDS. 